\title{
Analysis of candidate genes of spontaneous arthritis in mice deficient for interleukin-1 receptor antagonist
}

\author{
Yanhong $\mathrm{Cao}^{1,2}$, Caijuan $\mathrm{Li}^{3}$, Jian $\mathrm{Yan}^{4}$, Feng $\mathrm{Jiao}^{2}$, XiaoYun $\mathrm{Liu}^{2}$, Karen A. Hasty ${ }^{2}$, \\ John M. Stuart ${ }^{4}$, Weikuan $\mathrm{Gu}^{2 *}$ and Yan $\mathrm{Jiao}^{2 \dagger}$ \\ ${ }^{1}$ Institute of Kaschin-Beck Disease, Center for Endemic Disease Control, Centers for Disease \\ Control and Prevention, Harbin Medical University, Harbin, 150081, PR China \\ ${ }^{2}$ Departments of Orthopaedic Surgery-Campbell Clinic, and Pathology, University of \\ Tennessee Health Science Center (UTHSC), Memphis, TN 38163, USA \\ ${ }^{3}$ Department of Ultrasound, Hongqi Hospital of Mudanjiang Medical University, \\ Mudanjiang, Heilongjiang, 157011, PR China \\ ${ }^{4}$ Department of Medicine, University of Tennessee Health Science Center, \\ Memphis, TN 38163, USA
}

(Received 18 October 2011, accepted 14 February 2012)

\begin{abstract}
Previously, we identified a major quantitative trait locus (QTL) on mouse chromosome 1 that regulates the susceptibility to arthritis in an F2 population generated from arthritis-prone BALB/c and arthritis-resistant DBA/1 mice deficient for interleukin-1 receptor antagonist. To further select candidate genes for the QTL, we analyzed the expression patterns of arthritis in $38 \mathrm{~F} 2$ individuals and compared the expression levels of key candidate genes to the parental strains. Two distinct subpopulations of arthritic mice were identified in the 38 F2 mice. One subgroup of diseased mice was characterized by myeloid cell dominant inflammation, whereas the other was mainly associated with increased anti-apoptotic activities of inflammatory cells. Several differentially expressed important candidate genes in parental strains in the QTL region are relevant to myeloid cell, apoptotic activities, or to both. About one-quarter of those genes have been previously linked to arthritis in literature. The present study reveals two distinct subpopulations of arthritic mice with spontaneous arthritis due to deficiency for interleukin-1 receptor antagonist, suggesting that genes with function relevant to myeloid cell and/or apoptotic activities are most likely the key candidate genes for the QTL.
\end{abstract}

Key words: arthritis, candidate gene, microarray, mouse, QTL

\section{INTRODUCTION}

Rheumatoid arthritis (RA), like other common polygenic autoimmune diseases, is characterized by genetic risk factor make-up and phenotypic heterogeneity. Genetic polymorphisms of HLA genes, PTPN22, CTLA4, $P A D I 4, F c \gamma R s$, and various cytokine and cytokine-receptor loci have been reported in different RA populations by linkage and association studies (McInnes and Schett, 2007). In the clinic, fewer than half RA patients were positive in serum assays of the two most common RA markers, rheumatoid factors $(\mathrm{RFs})(28 \%)$ and anticyclic citrullinated peptide (anti-CCP) antibodies (40\%) at the time of diagnosis (van der Helm-van Mil et al., 2005). In

\footnotetext{
Edited by Toshihiko Shiroishi

* Corresponding author. E-mail: wgu@uthsc.edu

$†$ Corresponding author. E-mail: yjiao2@uthsc.edu

Note: Supplementary materials in this article are at http:// www.jstage.jst.go.jp/browse/ggs
}

response to TNF blocking therapy, most of RA patients show only partial or no improvement. Given the obvious heterogeneity, RA is sometimes regarded as a clinical syndrome, implying that complicated exclusive pathogenesis mechanisms are actually involved in different forms of RA. So it is appropriate to define precise classification criteria to identify different populations of RA patients to enable optimal treatment.

Traditionally, RA is defined by several clinical parameters, but there are no criteria available for RA subclassifications. Recently, microarray technology has proved effective in deciphering the biological and clinical diversity of cancer (Staudt, 2003). However, little, if any, effort has been made to subclassify RA patients or arthritic animals based on their individual baseline molecular variations. In the present study, we sought to subclassify the population of autoimmune arthritic mice due to the deficiency of interleukin-1 receptor antagonist (Il1rn) based on their gene expression patterns. 
BALB/c mice, but not other mouse strains, spontaneously develop RA-like autoimmune arthritis in the absence of Il1rn (Horai et al., 2000). Recent studies have confirmed that TNF and IL-17 play important roles in the pathogenesis of this disease (Horai et al., 2004; Nakae et al., 2003). Using an F2 population generated from arthritis-prone $\mathrm{BALB} / \mathrm{c}$ and arthritis-resistant DBA/1 mice deficient for $I l 1 r n$, we identified a major QTL on mouse chromosome 1 that is responsible for about $12 \%$ of the susceptibility to spontaneous arthritis (Jiao et al., 2011). To evaluate the candidate genes for the QTL, we analyzed the patterns of gene expression of 38 selected F2 individuals. We identified by sample clustering two subpopulations of arthritic mice with distinct gene expression patterns. Differentially expressed genes were also identified in different subpopulations of murine arthritis. We further examined the potential function of candidate genes selected according to the two patterns in the genomic QTL region.

\section{MATERIALS AND METHODS}

Mice BALB/c-based $\mathrm{Il} 1 \mathrm{rn}^{-/}$mice (KO) were kindly provided by Dr. Yoichiro Iwakura (Center for Experimental Medicine, Institute of Medical Science, University of Tokyo, Tokyo, Japan). They were bred to DBA/1 mice to generate DBA/1-based $I l 1 r^{-/-}$mice that were arthritis resistant. F1 mice were produced by crossing these two parental $\mathrm{Il} 1 \mathrm{rn}^{-1-}$ mice. F2 mice were then generated by intercrossing $\mathrm{F} 1$ hybrids. They were maintained at $22^{\circ} \mathrm{C}$ in the animal house of the Connective Tissue Research Center, University of Tennessee Health Science Center, Memphis, Tennessee, USA. The severity of arthritis was graded for each paw on a scale of 0 to 4 for the degree of redness and swelling (Sims et al., 2004). Male F2 mice with hind paw inflammation of 4 were randomly selected for microarray assays; nonarthritic F2 male mice were used as controls. Experiments were conducted according to the institutional ethical guidelines for animal experimentation and the safety guidelines for gene manipulation. The animal experiments were approved by the Committee for Animal Experiments of the Connective Tissue Research Center, University of Tennessee Health Science Center.

Total RNA isolation Total RNA was extracted using Trizol Reagent (Invitrogen, Trizol Reagent) according to the manufacturer's instructions. RNA quality was assessed using a Bioanalyser 2100 (Agilent, Santa Clara, $\mathrm{CA}$ ), and all the samples had a $28 \mathrm{~S} / 18 \mathrm{~S}$ ratio of $>1.8$. RNA was quantified using a Biophotometer (Eppendorf, Hauppauge, NY).

Affymetrix microarray assay Oligonucleotide microarray assays were carried out with beginning with $4 \mu \mathrm{g}$ of total RNAs by using the GeneChip system, including mouse genome 4302.0 arrays, one-cycle cDNA synthesis kit, sample cleanup module, IVT labeling kit, Fluidics Station 450, and Scanner 3000 (Affymetrix, Santa Clara, CA) according to the manufacturer's instructions. The assays were done individually for each sample. Raw data in CEL format were generated using GCOS 1.3 software (Affymetrix).

Microarray data analysis MAS5 method was applied to generate the detection calls (present, marginal, or absent). Raw intensity data were normalized using RMA algorithm with the Expression Console software (Affymetrix). Average perfect match (PM) mean intensity value across all the samples was chosen as filter during sample and gene clustering using dChip software (dChip: http://biosun1.harvard.edu/complab/dchip/). Comparative analysis was carried out between resultant arthritic and nonarthritic subclasses. Upon fold change equal to or greater than 1.5 filtering, statistical analysis was done to identify differentially expressed genes in diseased mice using EDGE tools (Leek et al., 2006) $(\mathrm{P}<$ 0.05). Functional clustering of these gene expression changes was carried out using DAVID tools $(P<0.01$ was considered significant) (Dennis et al., 2003).

Bioinformatic evaluation of function relevance to apoptotic and myeloid cells of candidate genes Evaluation of function of candidate genes was conducted with a searching tool, PGMapper (http://www.genediscovery. org/pgmapper/index.jsp) (Xiong et al., 2008). PGMapper identified all the possible candidate genes for the hypertension QTL by combining the mapping information from Ensemble database, updated literature information from PubMed, and the Online Mendelian Inheritance in Man (OMIM) database. For candidate genes, a potential connection with apoptotic inflammation and myeloid cells was evaluated by searching Online Mendelian Inheritance in Man (OMIM) (http://www.ncbi.nlm.nih.gov/ entrez/query.fcgi?db=OMIM) and PubMed (http://www. ncbi.nlm.nih.gov/entrez/query.fcgi?db=PubMed). Query terms were the combination of the name of the gene with any of these key words: apoptotic, inflammation, or myeloid cells. For any potential candidates, at least the abstract of one reference was read by two authors to determine a link between the gene and apoptotic and/or myeloid cells. For a gene with more than one reference that indicated its relevance, at least two references were read and cited in this study.

Data deposition The microarray data have been deposited in NCBI GEO database (accession no: GSE8690). 


\section{RESULTS}

Quality assessment of F2 arrays Raw data were generated and normalized using RMA algorithm with Expression Console software. To ensure proper inclusion of comparable arrays for our purpose, several param- eters, including housekeeping gene $3 / 5$ ratio, background intensity, and mean relative log expression signal (Fig. 1), were used to evaluate the overall quality of individual arrays, and four outlier arrays were discarded during further data mining.

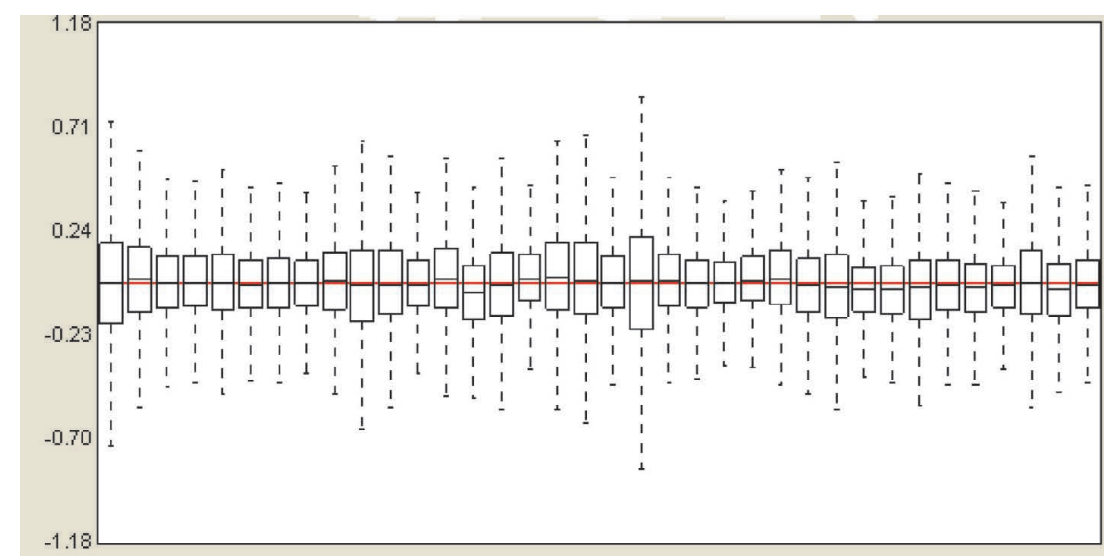

Fig. 1. Relative log splenic gene expression signals of F2 mice studied. Raw data microarray were normalized using RMA algorithm and visualized in a boxplot with Express Console (Affymetrix). The center line is the sample median value. All the studied samples showed similar medians.

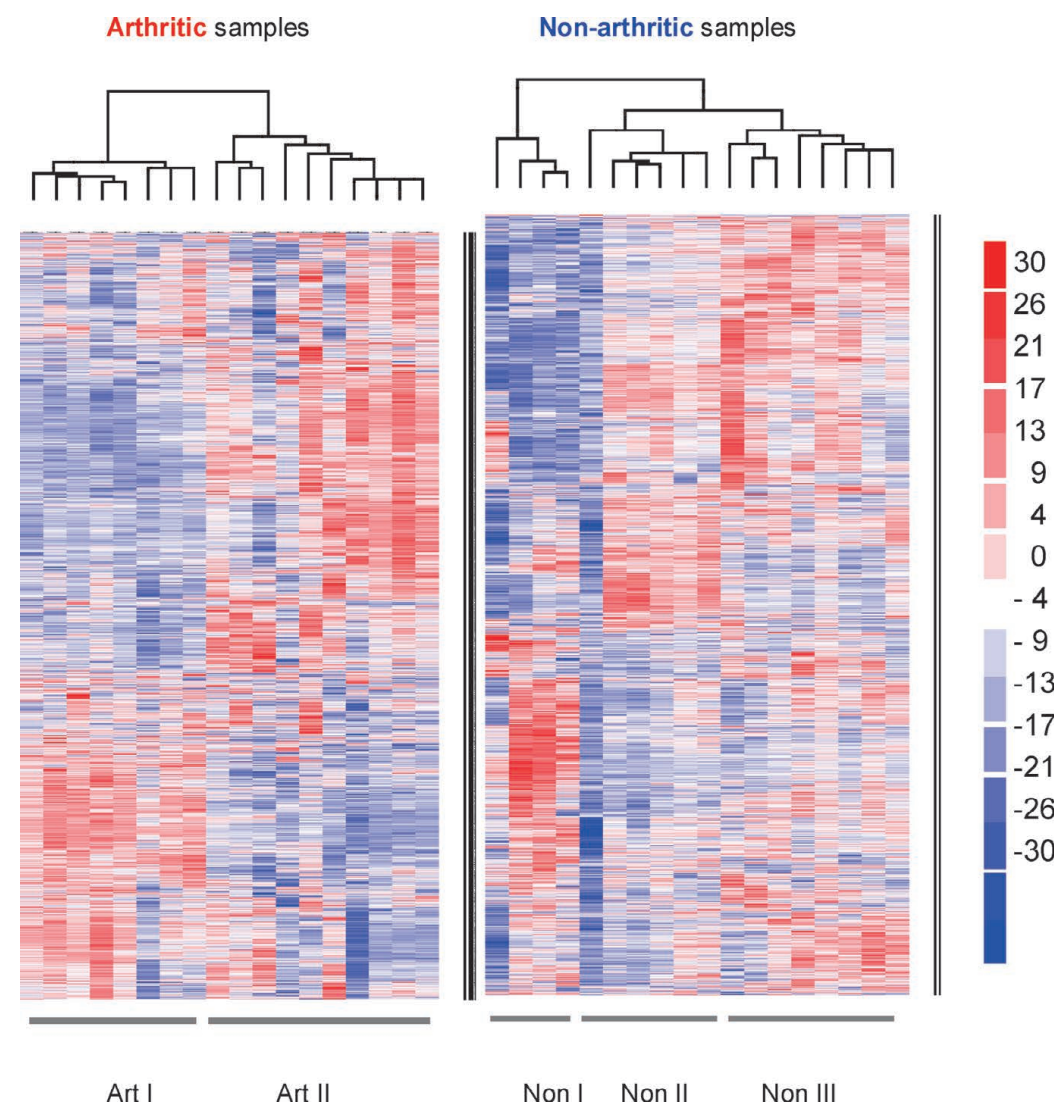

Fig. 2. Hierarchical clustering of samples from arthritic and nonarthritic F2 mice. Samples obtained from arthritic and nonarthritic mice were subject to hierarchical clustering separately using dChip software. Two subpopulations of arthritic mice and three subpopulations of nonarthritic mice were identified, respectively. Blue indicates lower-than-median signal. Red denotes higher-than-median signal. White represents equal-to-median signal. Scale bar is aligned on the right side of the figure. 
Table 1. Functional clustering of upregulated genes in Art 1 subgroup of arthritic mice

\begin{tabular}{|c|c|c|c|c|}
\hline Category & Count & $\mathrm{P}$ value & Genes & $\begin{array}{l}\text { known arthritis } \\
\text { relevant genes }\end{array}$ \\
\hline $\begin{array}{l}\text { alternative } \\
\text { splicing }\end{array}$ & 27 & 0.000 & $\begin{array}{l}\text { Incenp, Sept8, Polr3c, Bcl2l11, Rnf14, Fmr1, Ptdss2, } \\
\text { Cd44, Tmpo, Ighg, Epb4.1, Fbxo9, Zbp1, Dnm1l, Hipk1, } \\
\text { Ubtf, Slc4a1, Tsc22d3, Add2, Sox6, Eif4g1, Dpf3, Mxi1, } \\
\text { Mapk14, Ogfr, Hbs1l, AI314180, }\end{array}$ & $\begin{array}{l}\text { Cd44, Mapk14, } \\
\text { Eif4g1. }\end{array}$ \\
\hline atp-binding & 15 & 0.002 & $\begin{array}{l}\text { Hipk1, Rrm1, Dck, Camk2b, Mcm3, Hipk3, Atp2c1, } \\
\text { Abcb7, Wrn, Ercc6l, Atp8a1, Tlk1, Top2b, Mapk14, Kif11, }\end{array}$ & Mapk14 \\
\hline cell cycle & 15 & 0.001 & $\begin{array}{l}\text { Incenp, Spag5, Casp3, Camk2b, Mcm3, Aspm, Rbbp4, } \\
\text { Ccnb1-rs1, Sept8, Exo1, Tlk1, Mapk14, Ywhag, Kif11, Sesn1, }\end{array}$ & Mapk14, Casp3 \\
\hline $\begin{array}{l}\text { cellular protein } \\
\text { metabolism }\end{array}$ & 35 & 0.007 & $\begin{array}{l}\text { Spag5, Fbxw11, Usp7, Tfrc, Hipk3, St3gal2, Adam10, } \\
\text { Usp12, Irf7, Rnf14, Trim59, Epb4.2, Herc1, Tlk1, Herc5, } \\
\text { 9230105E10Rik, Mbtps1, Fbxo9, Hipk1, Pigq, Casp3, } \\
\text { Camk2b, Vps35, Otub2, Adamdec1, Oasl2, Eif4g1, Ibtk, } \\
\text { Mapk14, Hbs1l, Nktr, Parp12, Cebpb, Supt16h, Eif2s3x, }\end{array}$ & $\begin{array}{l}\text { Irf7, Adam10, } \\
\text { Cebpb, Mapk14, } \\
\text { Eif4g1, Casp3 }\end{array}$ \\
\hline DNA metabolism & 12 & 0.004 & $\begin{array}{l}\text { Hipk1, Exo1, Rrm1, Tlk1, Rad18, Top2b, Mcm3, Hipk3, } \\
\text { Wrn, Rbbp4, Tspyl1, Rrm2, }\end{array}$ & - \\
\hline immune response & 19 & 0.000 & $\begin{array}{l}\text { Arl6ip2, Casp3, Ifi203, Tlr13, Serpina3g, H28, Ifit3, Irf7, } \\
\text { Exo1, Oasl2, Ccl6, Ccl9, Clec4e, Mapk14, Tollip, Ccr2, } \\
\text { Fcgr1, Clec4d, Cebpb, }\end{array}$ & $\begin{array}{l}\text { Ccr2, Ccl9, } \\
\text { Cebpb, Tollip, } \\
\text { Mapk14, Ccl6, } \\
\text { Casp3 }\end{array}$ \\
\hline $\begin{array}{l}\text { intracellular } \\
\text { organelle }\end{array}$ & 59 & 0.009 & $\begin{array}{l}\text { Spag5, Tfrc, Hipk3, Arf2, St3gal2, Adam10, Wrn, Irf7, } \\
\text { Stat1, Fmr1, Epb4.2, Cyp20a1, Rad18, Kif11, Trak2, } \\
\text { Tmed2, Ugt8a, Ifi203, Mcm3, Serpina3g, Atp2c1, Abcb7, } \\
\text { Cxxc1, Ccnb1-rs1, Mxd1, Add2, Sox6, Exo1, Slc2a4, } \\
\text { Dpf3, Top2b, Mxi1, Steap3, Gusb, Cebpb, Parp12, } \\
\text { AI314180, Incenp, Dck, Usp7, Polr3c, Rnf14, Sec61a1, } \\
\text { Tlk1, Coro1c, Tmpo, Mbtps1, Hdlbp, Epb4.1, Hipk1, } \\
\text { Lancl2, Aspm, Ubtf, Rbbp4, Ap2a2, Phca, Mapk14, } \\
\text { Tspyl1, Sesn1, }\end{array}$ & $\begin{array}{l}\text { Stat1, Irf7, } \\
\text { Adam10, Mapk14, } \\
\text { Coro1c, Cebpb }\end{array}$ \\
\hline membrane & 35 & 0.000 & $\begin{array}{l}\text { Gp49a, Gna12, Clcn2, P2ry12, Tfrc, St3gal2, Adam10, } \\
\text { Bcl2l11, Sec61a1, Cyp20a1, Ptdss2, Tmpo, Clec4e, } \\
\text { Cd44, Ms4a6d, Mbtps1, Ccr2, Clec4d, Fcgr1, Vamp3, } \\
\text { Tmed2, Dnm1l, Pigq, Slc2a3, Lin7c, Ugt8a, Tlr13, Atp2c1, } \\
\text { Abcb7, Slc4a1, Add2, Atp8a1, Rtp4, Slc2a4, Phca, }\end{array}$ & $\begin{array}{l}\text { Cd44, ccr } 2, \\
\text { Clec } 4 d, \text { Adam } 10\end{array}$ \\
\hline nuclear protein & 33 & 0.000 & $\begin{array}{l}\text { Incenp, Dck, Hipk3, Irf7, Polr3c, Rnf14, Stat1, Fmr1, Tlk1, } \\
\text { Rad18, Tmpo, Hdlbp, Epb4.1, Hipk1, Lancl2, Ifi203, } \\
\text { Mcm3, Aspm, Serpina3g, Ubtf, Rbbp4, Cxxc1, Mxd1, } \\
\text { Sox6, Dpf3, Top2b, Mxi1, Mapk14, Cebpb, Parp12, } \\
\text { Tspyl1, AI314180, Sesn1, }\end{array}$ & $\begin{array}{l}\text { Stat1, Irf7, Cebpb, } \\
\text { Mapk14 }\end{array}$ \\
\hline $\begin{array}{l}\text { nucleic acid } \\
\text { binding }\end{array}$ & 35 & 0.005 & $\begin{array}{l}\text { Oas1a, Cpeb4, Wrn, Irf7, Polr3c, Ercc6l, Stat1, Fmr1, } \\
\text { Rad18, Carhsp1, Tmpo, Hdlbp, Zbp1, Rab18, Mcm3, } \\
\text { Ubtf, Cxxc1, Tsc22d3, Oas2, 4632434I11Rik, Mxd1, } \\
\text { Sox6, Exo1, Oasl2, Eif4g1, Dpf3, Pabpc1, Top2b, Mxi1, } \\
\text { Hbs1l, Supt16h, Cebpb, Parp12, Tspyl1, Eif2s3x, }\end{array}$ & $\begin{array}{l}\text { Stat1, Irf7, Oas2, } \\
\text { Cebpb, Eif4g1 }\end{array}$ \\
\hline phosphorylation & 25 & 0.000 & $\begin{array}{l}\text { Plek, Tfrc, Hipk3, Stat1, Epb4.2, Tlk1, Rad18, Carhsp1, } \\
\text { Cd44, Tmpo, Hdlbp, Epb4.1, Dnm1l, Hipk1, Casp3, } \\
\text { Camk2b, Mcm3, Atp2c1, Add2, Atp8a1, Eif4g1, Top2b, } \\
\text { Mapk14, Ywhag, AI314180, }\end{array}$ & $\begin{array}{l}\text { Cd44, Stat1, } \\
\text { Mapk14, Eif4g1, } \\
\text { Casp3 }\end{array}$ \\
\hline $\begin{array}{l}\text { pyrophosphatase } \\
\text { activity }\end{array}$ & 10 & 0.008 & $\begin{array}{l}\text { Dnm1l, Arl6ip2, Atp8a1, Gna12, Mcm3, Atp2c1, Abcb7, } \\
\text { Wrn, Nudt4, Ercc6l, }\end{array}$ & - \\
\hline transcription & 15 & 0.008 & $\begin{array}{l}\text { Hipk1, Mcm3, Hipk3, Ubtf, Rbbp4, Cxxc1, Irf7, Polr3c, } \\
\text { Mxd1, Rnf14, Stat1, Sox6, Dpf3, Mxi1, Cebpb, }\end{array}$ & Stat1, Irf7, Cebpb, \\
\hline transport & 15 & 0.007 & $\begin{array}{l}\text { Rab18, Clcn2, Slc2a3, Lin7c, Arf2, Atp2c1, Vps35, } \\
\text { Abcb7, Slc4a1, Ap2a2, Fmr1, Sec61a1, Slc2a4, Hdlbp, } \\
\text { Tmed2, }\end{array}$ & \\
\hline
\end{tabular}


Hierarchical clustering of samples To identify possible subpopulations of arthritic mice, we separately performed sample and gene clustering using PM mean intensity (500) (in 100\% of samples) filtered gene list on the arthritic subset of data. Two major subpopulations were identified in arthritic mice: Art I consisted of 8 samples, and Art II included 10 samples. Interestingly, three subpopulations of nonarthritic mice were also identified using this approach (Fig. 2). Although arthritis and non arthritis mice in the F2 population are grouped into separate clusters, those clusters are not completely separated. While many genes are overlapped among those groups, we indeed find some particular features of the arthritis groups (as shown below).

Molecular signatures of subpopulations of arthritic mice To characterize the molecular fingerprints of these two subpopulations of murine arthritis, we did comparative microarray analysis between two arthritic subgroups (Art I and Art II) and three nonarthritic subgroups of mice (Non I, Non II, and Non III). In general, 204 transcripts were found to be dysregulated in arthritic mice, thus changes of expression levels equal to or greater than 1.5-fold (Supplementary Table S1). Remarkably, $85 \%$ of transcripts were associated with arthritis in the Art I subpopulation, and only two transcripts (Csprs and an EST) were upregulated in both subpopulations.

In the Art I subpopulation, the expression levels of several genes that are involved in regulating inflammation (Adipoq, Fabp4, Dcn), angiogenesis (Nab2 and Ednrb), and $\mathrm{T}$ cell activation (Skap1) were lower than in nonarthritic mice. On the other hand, many genes (about 40) that are predominantly expressed in myeloid cells were upregulated (e.g., Ap2a2, Arf2, Carhsp1, Ccl6, Ccl9, Ccr2, Cd5l, Cebpb, Clec4d, Clec4e, Coro1c, Fcgr1, Gusb, Ifi203, Ifit3, Irf7, Lancl2, Ms4a6d, Oas1a, Oas2, Ogfr, P2ry12,
Phca, Pigq, Plek, Pmm2, Rnf14, Sec61a1, and Slfn4). Other prominent changes were the overexpression of many immunoglobulin genes (Ighg, Igk-V1, Igkv14-111, Igk-V21, Igk-V28, and Igkv6-23) and a $\mathrm{T}$ cell activation inhibitor, Camk2b. Functional annotation of the upregulated genes by DAVID tools revealed increased activity of cellular metabolism, cell cycle, transcription regulation, and immune response. The functional annotation of the upregulated genes indicated that a wide range of activities was stimulated, including transcriptional regulation, cell cycle, transport, immune response, and metabolism (Table 1).

In an attempt to see what percentage of those genes have been connected to arthritis in literature, we conducted a search of those genes and key term "arthritis" with our software PGMapper (Xiong et al., 2008). The data indicated that while linkage between arthritis and many genes in Table 1 have not been known, about a quarter of genes have been linked to arthritis in literature (Table 1).

In contrast, only a few genes were dysregulated in the Art II subpopulation, including mainly the downregulation of $C d 163$ and the upregulation of $B c l 2$ and $I l 7 r$.

Potential function in apoptotic and myeloid cells of key candidate genes in the QTL region In our previous study (Jiao et al., 2011), we found 11 genes (Mr1, Pla2g4a, Fasl, Prg4, Ptgs2, Tnfsf18, Tnfsf4, Rc3h1, Ncf2, Sell, and Selp) from the QTL region according to their differential expression. Using PGmapper, we conducted a search for their relevance to apoptotic and myeloid cells. Our data indicated that five genes (Fasl, Ptgs2, $M r 1, P l a 2 g 4 a$, and Prg4) are relevant to apoptotic inflammation while three (Fasl, Mr1, and Ptgs2) function in regulating myeloid cell-induced inflammation. These data affirmed our conclusion (Jiao et al., 2011) that Fasl, Mr1,

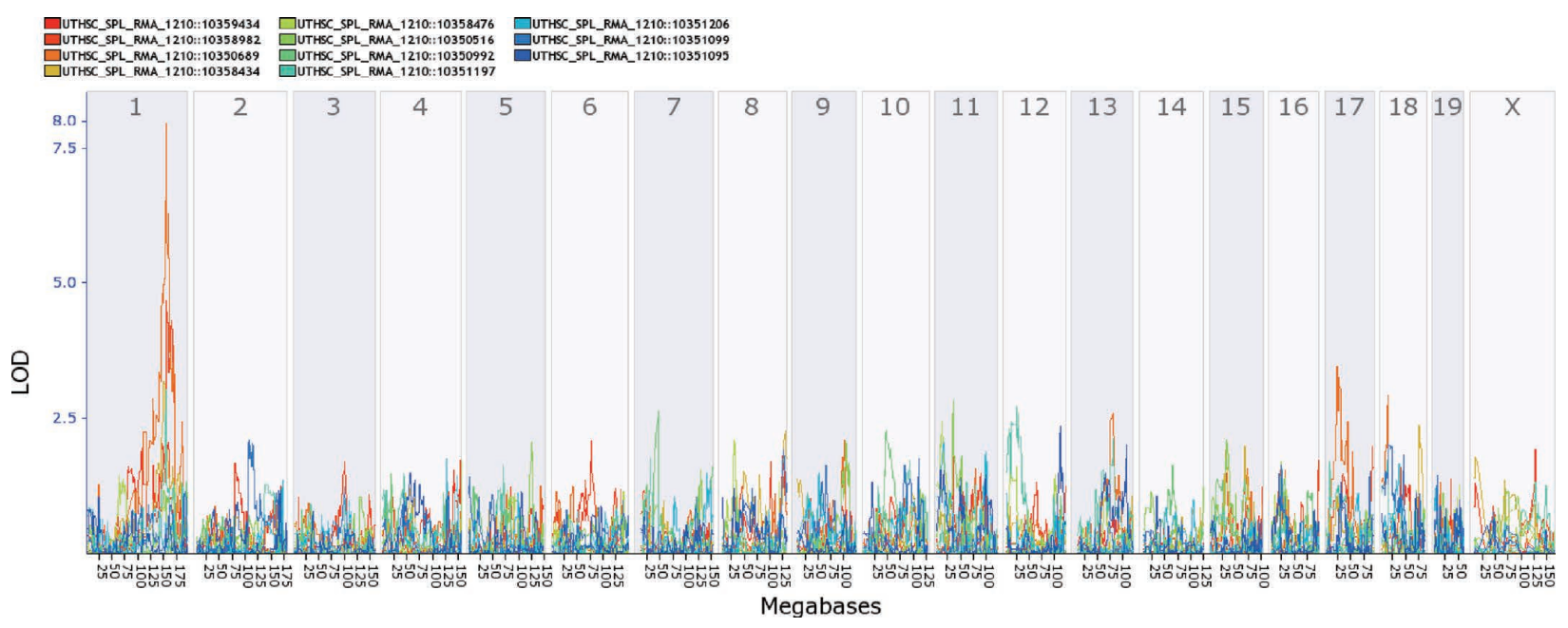

Fig. 3. Transcriptome mapping of 11 genes. Among 11 genes (Mr1, Pla2g4a, Fasl, Prg4, Ptgs2, Tnfsf18, Tnfsf4, Rc3h1, Ncf2, Sell, Selp), Pla2g4a was mapped on to chromosome 1, on which the QTL of arthritis is located. 
and Ptgs2 are the favorite candidate genes for the QTL.

In order to examine the genome regulation of those 11 genes, we further conducted transcriptome mapping of those 11 genes using gene expression profiles generated using the Affymetrix Gene Chip Mouse Gene 1.0 ST array (http://www.genenetwork.org/dbdoc/UTHSC_SPL_RMA_ 1210.html). Our analysis indicated that among those 11 genes, only Pla2g4a is mapped on a genomic region of chromosome 1, in which the QTL is also located (Fig. 3).

\section{DISCUSSION AND CONCLUSION}

Our data suggest two distinct subpopulations of spontaneous arthritis in the F2 mice based on their gene expression patterns. The expressions of key genes in these two subpopulation provide the basis for further testing in human populations. We feel that, because gene expression profiling technology was applied to F2 intercross mice, which bear a heterogeneous genetic background, the result fits the human population better than do homozygous strains. For example, in the Art I subpopulation, the altered gene expression pattern, with almost 40 myeloid genes being overexpressed, suggested the nature of myeloid cell dominance of arthritis. In the human population, the upregulated Fcgr1 is constitutively expressed on monocytes and macrophages (Rossman et al., 1989). Furthermore, its induced expression by synovial fluid neutrophils has been observed in RA patients (Quayle et al., 1997). Another upregulated gene, $C d 5 l$, which may play an important role in regulating adaptive immunity, is exclusively expressed by macrophages (Leek et al., 2006). Using DAVID tools, we found increased activity in transcriptional regulation, cell cycle, immune response, and metabolism (Table 1).

Immunoglobulin $(\mathrm{Ig})$ heavy $(\mathrm{H})$ and light $(\mathrm{L})$ chains contribute to autoimmune specificities (Radic et al., 1991). So a higher expression of a battery of $\mathrm{Ig} \mathrm{H}$ and $\mathrm{L}$ chain genes, including Ighg, Igk-V1, Igkv14-111, Igk-V21, Igk$V 28$, and Igkv6-23, may implicate the involvement of B lymphocyte activation. However, no evidence of $\mathrm{T}$ cell activation was found in this study. In contrast, a positive regulator of $\mathrm{T}$ cell receptor (TCR)-mediated signaling, Skap1 (Wu et al., 2002), was downregulated. The expression of Camk2b, which is a strong CD4 T cell inhibitor (Lin et al., 2005), was induced in extremely high abundance (5.29-fold increase). These findings suggest the possibility of alternative mechanisms for B cell activation.

As for the Art II subpopulation, the picture is quite different: only a few informative, differentially expressed genes were identified, including the downregulated Cd163 and upregulated Bcl2 and Il7r. $\quad C d 163$ is able to inhibit inflammation through mediating IL-10 release and heme oxygenase- 1 synthesis (Philippidis et al., 2004). Apoptosis of synovial macrophages, fibroblasts, and lymphocytes plays an important role in the development of RA (Liu and Pope, 2003). So the combined CD163 downregulation and upregulation of anti-apoptotic Bcl2 and Il7r (Jiang et al., 2004) may contribute greatly to the inflammatory process in the Art II subpopulation.

Perhaps the most important result in our study is the clarification or prioritizing of candidate genes for regulating the QTL on chromosome 1 that affect spontaneous arthritis in Babl/c mice of IL1rn deficiency. Previously, we listed several genes that are considered as candidates for the QTL genes (Jiao et al., 2011). Our current study suggests that, in addition to Fasl, Mr1, and Ptgs2, investigation of Pla2g4a is necessary, because, among 11 known candidate genes, its regulatory locus is the only one that is mapped into QTL region of spontaneous arthritis on chromosome 1 (Jiao et al., 2011).

The study was supported by grants from the National Institute of Arthritis and Musculoskeletal and Skin Diseases, National Institutes of Health (R01 AR51190 to WG; R01 AR50785 to JS), Project 81171679 supported by NSFC (to YHC), and the Veterans Administration Medical Center in Memphis, TN.

\section{REFERENCES}

Dennis, G., Jr., Sherman, B. T., Hosack, D. A., Yang, J., Gao, W., Lane, H. C., and Lempicki, R. A. (2003) DAVID: Database for Annotation, Visualization, and Integrated Discovery. Genome Biol. 4(5), P3.

Horai, R., Saijo, S., Tanioka, H., Nakae, S., Sudo, K., Okahara, A., Ikuse, T., Asano, M., and Iwakura, Y. (2000) Development of chronic inflammatory arthropathy resembling rheumatoid arthritis in interleukin 1 receptor antagonistdeficient mice. J. Exp. Med. 191, 313-320.

Horai, R., Nakajima, A., Habiro, K., Kotani, M., Nakae, S., Matsuki, T., Nambu, A., Saijo, S., Kotaki, H., Sudo, K., et al. (2004) TNF-alpha is crucial for the development of autoimmune arthritis in IL-1 receptor antagonist-deficient mice. J. Clin. Invest. 114, 1603-1611.

Jiang, Q., Li, W. Q., Hofmeister, R. R., Young, H. A., Hodge, D. R., Keller, J. R., Khaled, A. R., and Durum, S. K. (2004) Distinct regions of the interleukin-7 receptor regulate different Bcl2 family members. Mol. Cell. Biol. 24, 6501-6513.

Jiao, Y., Jiao, F., Yan, J., Xiong, Q., Shriner, D., Hasty, K., Stuart, J., and Gu, W. (2011) Identifying a major locus that regulates spontaneous arthritis in IL-1ra-deficient mice and analysis of potential candidates. Genet. Res. Camb. 93, 95-103.

Leek, J. T., Monsen, E., Dabney, A. R., and Storey, J. D. (2006) EDGE: extraction and analysis of differential gene expression. Bioinformatics 22, 507-508.

Lin, M. Y., Zal, T., Ch'en, I. L., Gascoigne, N. R., and Hedrick, S. M. (2005) A pivotal role for the multifunctional calcium/ calmodulin-dependent protein kinase II in T cells: from activation to unresponsiveness. J. Immunol. 174, 5583-5592.

Liu, H., and Pope, R. M. (2003) The role of apoptosis in rheumatoid arthritis. Curr. Opin. Pharmacol. 3, 317-322.

McInnes, I. B., and Schett, G. (2007) Cytokines in the pathogenesis of rheumatoid arthritis. Nature Reviews. Immunology 7, 429-442.

Nakae, S., Saijo, S., Horai, R., Sudo, K., Mori, S., and Iwakura, Y. (2003) IL-17 production from activated T cells is required 
for the spontaneous development of destructive arthritis in mice deficient in IL-1 receptor antagonist. Proc. Natl. Acad. Sci. USA 100, 5986-5990.

Philippidis, P., Mason, J. C., Evans, B. J., Nadra, I., Taylor, K. M., Haskard, D. O., and Landis, R. C. (2004) Hemoglobin scavenger receptor CD163 mediates interleukin-10 release and heme oxygenase- 1 synthesis: antiinflammatory monocyte-macrophage responses in vitro, in resolving skin blisters in vivo, and after cardiopulmonary bypass surgery. Circ. Res. 94, 119-126.

Quayle, J. A., Watson, F., Bucknall, R. C., and Edwards, S. W. (1997) Neutrophils from the synovial fluid of patients with rheumatoid arthritis express the high affinity immunoglobulin G receptor, Fc gamma RI (CD64): role of immune complexes and cytokines in induction of receptor expression. Immunology 91, 266-273.

Radic, M. Z., Mascelli, M. A., Erikson, J., Shan, H., and Weigert, M. (1991) Ig H and L chain contributions to autoimmune specificities. J. Immunol. 146, 176-182.
Rossman, M. D., Chen, E., Chien, P., Rottem, M., Cprek, A., and Schreiber, A. D. (1989) Fc gamma receptor recognition of IgG ligand by human monocytes and macrophages. Am. J. Respir. Cell Mol. Biol. 1, 211-220.

Sims, N. A., Green, J. R., Glatt, M., Schlict, S., Martin, T. J., Gillespie, M. T., and Romas, E. (2004) Targeting osteoclasts with zoledronic acid prevents bone destruction in collageninduced arthritis. Arthritis Rheum. 50, 2338-2346.

Staudt, L. M. (2003) Molecular diagnosis of the hematologic cancers. N. Engl. J. Med. 348, 1777-1785.

van der Helm-van Mil, A. H., Wesoly, J. Z., and Huizinga, T. W. (2005) Understanding the genetic contribution to rheumatoid arthritis. Curr. Opin. Rheumatol. 17, 299-304.

Wu, L., Fu, J., and Shen, S. H. (2002) SKAP55 coupled with CD45 positively regulates T-cell receptor-mediated gene transcription. Mol. Cell. Biol. 22, 2673-2686.

Xiong, Q., Qiu, Y., and Gu, W. (2008) PGMapper: a web-based tool linking phenotype to genes. Bioinformatics 24, 10111013. 